\title{
The Study of Reducing Catatonic Features by Occupational Therapy Intervention
}

\author{
M. Ramakrishnan, K. Kalaichandran, R. Ilavajagan \\ MOT (mental health), Occupational therapist, department of Psychiatry, JIPMER Hospital, Puducherry-6, \\ India, \\ M.O.T (Neuro), MSc (Psy), MSc (App Psy) Senior Occupational Therapist, Raja Muthiah Medical College \\ Hospital, Annamalai University \\ Former, Professor\& Head, Faculty of Health Science, Kuala Lumpur Metropolitan University (KLMU), Kuala \\ Lumpur, Malaysia \\ MOT (mental health), MD (Ac.), Director-Arisjuvady Mental Health centre, Puducherry-7, India,
}

\begin{abstract}
:
Background study: Schizophrenia is a serious mental illness that affects one person in a hundred at some stage in life (Scottish Intercollegiate Guidelines Network [SIGN], 1998). Initial onset is usually in the teens or twenties and the subsequent course is variable. Unless the initial episode is brief, incomplete recovery and further relapses are the most likely outcome (SIGN, 1998). Rehabilitation Rehabilitation emphasizes social and vocational training to help people with schizophrenia function better in their communities. Because schizophrenia usually develops in people during the critical career-forming years of life (ages 18 to 35), and because the disease makes normal thinking and functioning difficult, most patients do not receive training in the skills needed for a job. Rehabilitation programs can include job counseling and training, money management counseling, help in learning to use public transportation, and opportunities to practice communication skills. Rehabilitation programs work well when they include both job training and specific therapy designed to improve cognitive or thinking skills. Programs like this help patients hold jobs, remember important details, and improve their functioning.
\end{abstract}

\section{I.Introduction}

Schizophrenia can also begin later in life (e. g., after age 45 years), but this is uncommon. Usually the onset of Schizophrenia occurs a few years earlier in men than women. The onset may be abrupt or insidious. Usually Schizophrenia starts gradually with a pre psychotic phase of increasing negative symptoms (e. g., social withdrawal, deterioration in hygiene and grooming, unusual behavior, outbursts of anger, and loss of interest in school or work). A few months or years later, a psychotic phase develops (with delusions, hallucinations, or grossly bizarre/disorganized speech and behavior). Individuals who have an onset of Schizophrenia later in their 20 's or 30's are more often female, have less evidence of structural brain abnormalities or cognitive impairment, and display a better outcome. Schizophrenia usually persists, continuously or episodically, for a lifetime. Complete remission (i. e., a return to full pre morbid functioning) is uncommon. Some individuals appear to have a relatively stable course, whereas others show a progressive worsening associated with severe disability. The psychotic symptoms usually respond to treatment with anti psychotic medication, whereas the negative symptoms are less responsive to anti psychotic medication. Often the negative symptoms steadily become more prominent during the course of Schizophrenia. F20.2 Catatonic Schizophrenia Catatonic Schizophrenia is dominated by prominent psycho motor disturbances that may alternate between extremes such as hyper kinesis and stupor, or automatic obedience and negativism. Constrained attitudes and postures may be maintained for long periods. Episodes of violent excitement may be a striking feature of the condition. The catatonic phenomena may be combined with a dream-like (oneiroid) state with vivid scenic hallucinations.

\section{Cognitive Impact}

The use of cognitive tasks and concepts in schizophrenia has grown significantly in recent years, and has revealed cognition as being of utmost importance in the study, treatment and long-term management of the disease (Heinrichs, 2005). Cognitive test scores are now considered more accurate than symptomatology measures as predictors of community functioning (Green, 1996; Velligan et al., 1996). Further, recent metaanalyses have shown specific measures of cognition (particularly tests of set-shifting, verbal fluency, and sustained attention) are more sensitive to differences between schizophrenia and healthy participants than brain imaging or postmortem examinations (Heinrichs, 2005). Finally, impaired cognition precedes, accompanies and outlasts a patient's symptoms and medical regimen (Heinrichs, 2005), so its influence cannot be underestimated. 


\section{Psychosocial Interventions}

A recent study reported the impact of pharmacological treatments for schizophrenia is modest at 25 percent improvement over no treatment (World Health Organization, 2005). However, the addition of psychosocial treatment increased the rate of improvement to $45 \%$. Many recent studies encourage an integration of pharmacological and psychosocial interventions in the treatment of schizophrenia. For example, Kopelowicz and Liberman (2003), state the use of drugs to manage symptoms requires concurrent psychosocial interventions to improve involvement and compliance in treatment, and obtain optimal long-term therapeutic outcomes. Foulds (2006) suggests the use of psychosocial interventions should be considered consistently alongside medication in order to manage symptoms and encourage reality testing. The Clinical Resource and Audit Group (CRAG) identified the following as goals for psychosocial therapies for individuals with schizophrenia: assessment, support, education, increasing concentration, reality reinforcement, improved communication and relationship skills, anxiety and mood management, daily living skills, and time management (as cited in SIGN, 1998). The accumulation of evidence for effective psychosocial interventions such as social skills training, family therapy and vocational rehabilitation, has helped to raise awareness of the need for such services, thus broadening the treatment options for people with schizophrenia (West et al, 2005). Bustillo, Lauriello, Horan and Keith (2001) reviewed randomized controlled trials, with particular emphasis on those published since 1996. They identified 18 new studies: five for cognitive behaviour therapy, five for social skills training, three for supported employment programs, two for family therapy, two for case management, and 14 one for individual therapy. They found the interventions have been largely successful for the primary outcome measure they were intended to address. As a result of their review, Bustillo and colleagues (2001) recommend psycho educational family interventions for those patients who live with family members, assertive community treatment programs for patients with high service utilization, a systemic psychosocial rehabilitation and social skills education plan for those living in the community, vocational rehabilitation for those patients who wish to work, and cognitive behavioural interventions to assist with ongoing delusions and hallucinations. Practice guidelines, algorithms and treatment manuals promoting integrated therapy for schizophrenia have been published by several leading mental health organizations; however, evidence suggests that most clinicians are not following these guidelines (Liberman \& Glick, 2004). It has been argued there is little training for psychiatrists in integrating treatments, leaving the focus of treatment on the pharmacologic aspect, and neglecting the importance of psychosocial rehabilitation in recovery. Liberman \& Glick (2004) suggest an even darker reason: Competencies [of new psychiatrists] include checking of the criterion symptoms for establishing a DSM-IV diagnosis and prescribing medications that have been compellingly promoted by drug companies. Many academics, who teach in continuing education programs that are sponsored by the pharmaceutical industry, while ostensibly providing scientific and evidence-based information, are inevitably biased by their handsome consulting honoraria (p.1217). While this paper is not the medium through which to debate the long arm of the pharmaceutical industry, it is important to acknowledge the unmet need for psychosocial services among individuals with schizophrenia. A 1998 study found as few as 10\% of mental health patients received psychosocial interventions (Lehman \& Steinwachs, 1998). This situation was echoed in a later U.S. study by West and colleagues (2005), 15 which assessed national conformance with practice guideline treatment recommendations of the Schizophrenia Patient Outcomes Research Team and the American Psychiatric Association. They found rates of conformance for psychopharmacologic recommendations were relatively high $(30-100 \%)$ whereas rates for psychosocial recommendations were significantly lower $(0-43 \%)$ (p. 287). Dhillon and Dollieslager (2000), as well as Torrey and colleagues (2001) identified barriers to implementing evidence-based psychosocial rehabilitation programs, such as a history of emphasis on biological treatment; the belief of staff that acutely ill patients cannot participate in psychosocial rehabilitation; a lack of accountability for providing psychosocial interventions; the wide range of symptoms and levels of functioning presented by patients; and clinicians' resistance to change. However, these obstacles were overcome through the use of welldesigned training tools such as workbooks, clinical observation and supervision, and consultation materials; and accountability was ensured through the use of fidelity measures and feedback mechanisms (Torrey et al., 2001). Consequently, two significant outcomes were achieved. First, highly structured and effective evidence-based psychosocial treatment plans were successfully implemented in the respective clinical settings. Secondly, implementation plans and best practice guidelines were developed, which can assist other psychosocial rehabilitation clinicians to provide evidence-based best practice interventions.

\section{II.Case Description}

This descriptive case study illustrates the experience of a 46 years old male with catatonia, clinical features of highly stupor, waxy flexibility with mute behavior and not listens to family members and the hospital staff, poor self-care, poor physical activity, poor communication and poor socio occupational functioning. He was married and having children. His wife role is housewife. He finished degree and joined military, due to family issues such as wife does not take care of home and she wants her husband to take family 
responsibility so he got voluntary retirement then he start to take a family responsibilities with his pension amount. He became stoop and upset, falls in to mental illness. Family members took him to various hospitals for the treatment; meanwhile, his wife was not supporting him in the life. He has the medication except rehabilitation. Finally, he brought to JIPMER Hospital by his father for his remedy. Here he seems to be very gigantic figure but poor physical activity. He was admitted for more than two months for his improvement. He started medication and planned for to give ten ECT but they given only two ECT. Then he referred to the occupational therapy for the rehabilitation.

\section{III.Occupational Therapy Management}

On observation he seems to be stout and fully shaved, he found to be mute and highly stoop posture and wax flexibility. So the therapist cannot interview him initially, the data was collected from his father and the brother-in-law. After two weeks, he brought to the occupational therapy department by his father along with the therapist for the rehabilitation. Then he was examined. And he was planned for activity scheduling. In the scheduling activities are water coloring, paintings and throw ball game. In the occupational department he can occupy more interested only playing table tennis on one to one basis in the both sessions. He played well because this only he previously experienced when he was normal in his working place. Therapy sessions are one hour in the morning, two hours in the evening/Day, 24 hours/Week for 12 Weeks. Rest of the period he had been practiced walking exercise. He got verbal prize and gave the tea token as a positive reinforcement by the occupational therapist for the succeeding the table tennis. He was kept with fully shaved as a robotic movement. He was changed his face by not removing the mustache and shaving the beard like his father. Here it was found that he changed his facial expression and start doing the all the activities by own with surprisingly only medication being continued for the illness.

\section{IV.Discussion}

In this case self-concept which includes self-esteem, self-confidence and self-actualization are developed slowly through conscious and sub conscious. There was seen that no disturbance in sleeping pattern. He might be perceiving his face by self with good and appearance well. Basically he comes from village, brought up traditional pattern and his father has big mustache so it may be the one of the reason for his improvement. In the therapy side he was given positive reinforcement and conduct indoor game for him, through these can show this improvement also, occupational therapy goal is make him independent in the area of self-care, play/leisure and work/task. Before the rehabilitation there was no facial expression until the perceiving of his own face by self. After the therapy he starts little bit initiation in smiling face, self-care and the simple task and also prevents pre occupation.

\section{V.Conclusion}

Occupational Therapy helps patient with catatonic features to be in contact with tradition and the inner mind. The findings of the study are consistent with the assumption. These suggest that the catatonic patient has improved by medicine along with occupational therapy intervention.

$\begin{array}{ll}\text { Ethical Clearance: } & \text { NIL } \\ \text { Conflict Of Interest: } & \text { NIL } \\ \text { Source of Funding: } & \text { NIL }\end{array}$

\section{References}

[1]. Schizophrenia - DSM-IV Diagnostic Criteria, American Psychiatric Association

[2]. Addington, D., Boouchard, R., Goldberg, J., Honer, B., Malla, A., Norman, R., Tempier, R., Berzins, S. (2005). Clinical practice guidelines: Treatment of schizophrenia. Canadian Journal of Psychiatry, 50, 1.

[3]. Bustillo, J., Lauriello, J., Horan, W., \& Keith, S. (2001). The psychosocial treatment of schizophrenia: An update. American Journal of Psychiatry, 158, 163-175.

[4]. Cohen, A., Forbes, C., Mann, M., \& Blanchard, J. (2006). Specific cognitive deficits and differential domains of social functioning impairment in Schizophrenia. Schizophrenia Research, 81, 227-238.

[5]. Lehman, A., \& Steinwachs, D. (1998). Patterns of usual care for schizophrenia: initial results from the Schizophrenia Patient Outcomes Research Team (PORT) client survey. Schizophrenia Bulletin, 24, 11-20.

[6]. Liberman, R., \& Glick, I. (2004). Drug and psychosocial curricula for psychiatry residents for treatment of schizophrenia: Part I. Psychiatric Services, 55, 11, 1217-1218.

[7]. Torrey, W., Drake, R., DIXON, L., Burns, B., Flynn, L., Rush, J., et al. (2001). Implementing evidence-based practices for persons with severe mental illnesses.

[8]. World Health Organization. (2005). choosing cost-effective interventions in psychiatry: results from the CHOICE programme of the World Health Organization. World Psychiatry 4, 1, 37-44.

[9]. West, J., Wilk, J., Olfson, M., Rae, D., Marcus, S., Narrow, et al. (2005). Patterns and quality of treatment for patients with schizophrenia in routine psychiatric practice.

[10]. Willard \& Spackman (1993) eight edition Willard \& Spackman's Occupational Therapy. 